\title{
Chebyshev polynomials for approximation of solution of fractional partial differential equations with variable coefficients
}

\author{
Yang Zhongshu ${ }^{1, \mathrm{a}}$ and Zhang Hongbo ${ }^{1, \mathrm{~b},{ }^{*}}$ \\ ${ }^{1}$ Naval aeronautical engineering application, Beijing, 100071, China \\ ayangzhongshu01@163.com, 'zhanghongbo90@163.com
}

Keywords: Chebyshev polynomials, fractional partial differential equation with variable coefficients, operational matrix, numerical solution.

Abstract. In this paper, a numerical method for solving a class of fractional partial differential equations with variable coefficients based on Chebyshev polynomials is proposed. The fractional derivative is described in the Caputo sense. The properties of Chebyshev polynomials are used to reduce the initial equations to the products of several matrixes. A system of linear equations are obtained by dispersing the coefficients and the products of matrixes. Only a small number of Chebyshev polynomials are needed to acquire a satisfactory result. Results obtained using the scheme presented here show that the numerical method is very effective and convenient for solving fractional partial differential equations with variable coefficients.

\section{Introduction}

In recent years, fractional derivative and fractional differential equations have found their applications in different disciplines. A lot of practical problems can be elegantly modeled with the help of the fractional derivative. For example, nonlinear oscillation of earthquake may be modeled with fractional derivatives [1], many other recent developments in the description of anomalous transport by fractional dynamics [2]. Due to the increasing applications, a lot of attention has been paid to numerical and exact solution of fractional differential equations and fractional partial equations. The analytical solutions of fractional differential equations are still in a preliminary stage. However, it is difficult to obtain their exact solutions. Both mathematicians and physicists have engaged in discussing the numerical methods for solving fractional differential equations. The most commonly used ones are Adomian decomposition method (ADM) [3,4], Variational iteration method (VIM) [5], Generalized differential transform method (GDTM) [6-8], Finite Difference Method (FDM) [9] and Wavelet method $[10,11]$ and so on.

In this paper, our study focuses on a class of fractional partial differential equation as following:

$a(x) \frac{\partial^{\alpha} u(x, t)}{\partial x^{\alpha}}+b(x) \frac{\partial^{\beta} u(x, t)}{\partial t^{\beta}}=f(x, t)$,

subject to the initial conditions

$u(x, 0)=g(x)$

$u(0, t)=h(t)$

where $\partial^{\alpha} u(x, t) / \partial x^{\alpha}$ and $\partial^{\beta} u(x, t) / \partial t^{\beta}$ are fractional derivative of Caputo sense, $0<\alpha, \beta \leq 1$, $f(x, t), a(x)$ and $b(x)$ are the known and $u(x, t)$ is unknown.

There have been several methods for solving the fractional partial differential equation. Odibat used successfully generalized differential transform method to solve the numerical solution of linear partial differential equations of fractional order [12].Yi et.al. [13] applied block pulse functions method to obtain the fractional partial equations. Podlubny solved the fractional partial differential equations with constant coefficients by using Laplace Transform method .

The structure of this paper is as follows: In Section 2, basic fractional derivatives and integrals definitions are reviewed. The basic definitions of Chebyshev polynomials function approximation is given in Section 3. In Section 4, the fractional operational matrix of Chebyshev polynomials are derived. 
We also apply the operational matrix to solve the fractional partial differential equations in Section 4. Numerical examples are provided to clarify the approach in Section 5. We end the paper with concluding remarks.

\section{Definitions of fractional derivatives and integrals}

Riemann-Liouville fractional integral of order $\alpha,(\alpha \geq 0)[14]$

$$
I_{t}^{\alpha} u(t)=\frac{1}{\Gamma(\alpha)} \int_{0}^{t}(t-\tau)^{\alpha-1} u(\tau) d \tau, t>0
$$

The properties of the Riemann-Liouville fractional integral can be given by:

(i) $I^{\alpha} I^{\beta} u(x)=I^{\beta} I^{\alpha} u(x)$

(ii) $I^{\alpha} I^{\beta} u(x)=I^{\alpha+\beta} u(x)$

Riemann-Liouville fractional derivative of order $\alpha,(\alpha \geq 0)$ [14]

$$
D_{t}^{\alpha} u(t)=\left\{\begin{array}{lc}
\frac{d^{r} u(t)}{d t^{r}} & \alpha=\mathbf{R} \in \mathbf{N} \\
\frac{1}{\Gamma(r-\alpha)} \frac{d^{r}}{d t^{r}} \int_{0}^{t} \frac{u(\tau)}{(t-\tau)^{\alpha-r+1}} d \tau, & 0 \leq r-1<\alpha<r
\end{array}\right.
$$

Caputo's fractional derivative of order $\alpha,(\alpha \geq 0)$ [ 14]

$$
D_{*}^{\alpha} u(t)=\left\{\begin{array}{lc}
\frac{d^{r} u(t)}{d t^{r}} & \alpha=\mathbf{R} \in \mathbf{N} \\
\frac{1}{\Gamma(r-\alpha)} \int_{0}^{t} \frac{u^{r}(\tau)}{(t-\tau)^{\alpha-r+1}} d \tau, & 0 \leq r-1<\alpha<r
\end{array}\right.
$$

Specially, for the Caputo derivative, we have ( $c$ is a constant)

$D_{*}^{\alpha} c=0$,

$$
D_{*}^{\alpha} x^{\beta}=\left\{\begin{array}{l}
0, \quad \beta \in \mathbf{N}_{0}, \beta<\lceil\alpha\rceil . \\
\frac{\Gamma(\beta+1)}{\Gamma(\beta+1-\alpha)} x^{\beta-\alpha}, \beta \in \mathbf{N}_{0}, \beta \geq\lceil\alpha\rceil ; \beta \notin \mathbf{N}, \beta>\lfloor\alpha\rfloor .
\end{array}\right.
$$

The relationship between the Riemann-Liouville operator and Caputo operator is expressed as the following.

(i) $D_{*}^{\alpha} I^{\alpha} u(t)=u(t)$

(ii) $I^{\alpha} D_{*}^{\alpha} u(t)=u(t)-\sum_{k=0}^{r-1} u^{(k)}\left(0^{+}\right) \frac{t^{k}}{k !}, t>0$

\section{Chebyshev polynomials and some of their properties}

The well known Chebyshev polynomials are defined on the interval $[-1,1]$ and can be determined with the recurrence formula [15] 


$$
T_{n+1}(x)=2 x T_{n}(x)-T_{n-1}(x), \quad T_{0}(x)=1, T_{1}(x)=x, \quad n=1,2, \mathrm{~K},
$$

The analytic form of the Chebyshev polynomials $T_{n}(x)$ of degree $n$ is given by

$T_{n}(x)=n \sum_{i=0}^{\left[\frac{n}{2}\right]}(-1)^{i} 2^{n-2 i-1} \frac{(n-i-1) !}{(i) !(n-2 i) !} x^{n-2 i}$

where $[n / 2]$ denotes the integer part of $n / 2$. The orthogonality condition is

$$
\int_{-1}^{1} \frac{T_{i}(x) T_{j}(x)}{\sqrt{1-x^{2}}} d x= \begin{cases}\pi, & i=j=0 \\ \pi / 2, & i=j \neq 0 . \\ 0, & i \neq j\end{cases}
$$

In order to use these polynomials on the interval $[0,1]$, we define the shifted Chebyshev polynomials by introduce the change of variable $x=2 t-1$. Therefore, the shifted Chebyshev polynomials are defined as $T_{n}^{*}(t)=T_{n}(2 t-1)$. The analytic form of the shifted Chebyshev polynomials $T_{n}^{*}(t)$ of degree $n$ is given by

$T_{n}^{*}(t)=n \sum_{k=0}^{n}(-1)^{n-k} \frac{2^{2 k}(n+k-1) !}{(2 k) !(n-k) !} t^{k}, \quad n=1,2, \mathrm{~K}$

Let

$\boldsymbol{\Phi}(t)=\left[T_{0}^{*}(t), T_{1}^{*}(t), \mathrm{L}, T_{n}^{*}(t)\right]^{\mathrm{T}}$

The Chebyshev polynomials given by Eq.(11) can be expressed in the matrix form

$\boldsymbol{\Phi}(t)=\boldsymbol{A} \Delta_{n}(t)$

where

$\boldsymbol{A}=\left[\begin{array}{ccccc}1 & 0 & 0 & \mathrm{~L} & 0 \\ -1 & 2 & 0 & \mathrm{~L} & 0 \\ 2(-1)^{2} \frac{1 !}{2 !} & 2(-1)^{1} \frac{2^{2} 2 !}{2 !} & 2(-1)^{0} \frac{2^{4} 3 !}{4 !} & \mathrm{L} & 0 \\ \mathrm{M} & \mathrm{M} & \mathrm{M} & \mathrm{O} & \mathrm{M} \\ n(-1)^{n} \frac{(n-1) !}{n !} & n(-1)^{n-1} \frac{2^{2} n !}{2 !(n-1) !} & n(-1)^{n-2} \frac{2^{4}(n+1) !}{4 !(n-2) !} & \mathrm{L} & n(-1)^{0} \frac{2^{2 n}(2 n-1) !}{(2 n) !}\end{array}\right]$,

$\Delta_{n}(t)=\left[1, t, \mathrm{~K}, t^{n}\right]^{T}$

Obviously

$\Delta_{n}(t)=\mathbf{A}^{-1} \Phi(t)$

A function $u(t) \in L^{2}(0,1)$ can be expressed in terms of the Chebyshev basis. In practice, only the first $(n+1)$ term of Chebyshev polynomials are considered. Hence

$u(t) \cong \sum_{i=0}^{n} c_{i} T_{i}^{*}(t)=c^{\mathrm{T}} \boldsymbol{\Phi}(t)$

where $c=\left[c_{0}, c_{1}, \mathrm{~L}, c_{n}\right]^{\mathrm{T}}, c_{i}(i=0,1,2, \mathrm{~L}, n)$ are called Chebyshev coefficients, and 
For the function $u(x, t) \in L^{2}([0,1] \times[0,1])$, we can also obtain its approximation by using Chebyshev polynomials

$u(x, t) \cong \sum_{i=0}^{n} \sum_{j=0}^{n} u_{i j} T_{i}^{*}(t) T_{j}^{*}(t)=\boldsymbol{\Phi}^{\mathrm{T}}(x) \boldsymbol{U} \boldsymbol{\Phi}(t)$

where $\boldsymbol{U}=\left[\begin{array}{cccc}u_{00} & u_{01} & \mathrm{~L} & u_{0 n} \\ u_{10} & u_{11} & \mathrm{~L} & u_{1 n} \\ \mathrm{M} & \mathrm{M} & \mathrm{O} & \mathrm{M} \\ u_{n 0} & u_{n 1} & \mathrm{~L} & u_{n n}\end{array}\right]$

Theorem 1. [15] The error in approximating $u(t)$ by the sum of its first $n$ terms is bounded by the sum of the absolute values of all the neglected coefficients. If

$u_{n}(t)=\sum_{i=0}^{n} c_{i} T_{i}^{*}(t)$

then

$E_{T}(m)=\left|u(t)-u_{n}(t)\right| \leq \sum_{k=n+1}^{\infty}\left|c_{k}\right|$

for all $u(t)$, all $n$, and all $t \in[-1,1]$.

Theorem 2. [16] The Caputo fractional derivative of order $\alpha$ for the shifted Chebyshev polynomials can be expressed in terms of the shifted Chebyshev polynomials themselves in the following form $D^{\alpha}\left(T_{i}^{*}(t)\right)=\sum_{k=\lceil\alpha\rceil}^{i} \sum_{j=0}^{k-\lceil\alpha\rceil} \theta_{i, j, k} T_{j}^{*}(t)$

where $\theta_{i, j, k}=\frac{(-1)^{i-k} 2 i(i+k-1) ! \Gamma\left(k-\alpha+\frac{1}{2}\right)}{\Gamma\left(k+\frac{1}{2}\right)(i-k) ! \Gamma(k-\alpha-j+1) \Gamma(k-\alpha+j+1)}, \quad j=0,1, \mathrm{~K}$.

Theorem 3. The error $E_{T}^{*}(m)=\left|D^{\alpha}(u(t))-D^{\alpha}\left(u_{n}(t)\right)\right|$ in approximating $D^{\alpha}(u(t))$ by $D^{\alpha}\left(u_{n}(t)\right)$ is bounded by $\left|E_{T}^{*}(m)\right| \leq \mid \sum_{i=n+1}^{\infty} c_{i}\left(\sum_{k=\lceil\alpha\rceil}^{i} \sum_{j=0}^{k-\lceil\alpha\rceil} \theta_{i, j, k}\right)$.

Proof. A combination of Eq.(18) and (23) leads to

$$
\begin{aligned}
\left|E_{T}^{*}(m)\right| & =\left|D^{\alpha}(u(t))-D^{\alpha}\left(u_{n}(t)\right)\right| \\
& =\mid \sum_{i=n+1}^{\infty} c_{i}\left(\sum_{k=\lceil\alpha\rceil}^{i} \sum_{j=0}^{k-\lceil\alpha\rceil} \theta_{i, j, k} T_{j}^{*}(t)\right)
\end{aligned}
$$

but $\left|T_{j}^{*}\right| \leq 1$, so we can get

$$
\left|E_{T}^{*}(m)\right| \leq \mid \sum_{i=n+1}^{\infty} c_{i}\left(\sum_{k=\lceil\alpha\rceil}^{i} \sum_{j=0}^{k-\lceil\alpha\rceil} \theta_{i, j, k}\right),
$$

and subtracting the truncated series from the infinite series, bounding each term in the difference, summing the bounds and hence completes the proof of the theorem. 


\section{Numerical solution of the fractional partial differential equation with variable coefficients}

Consider the fractional partial differential equation with variable coefficients Eq. (1). If we approximate the function $u(x, t)$ with the Chebyshev Polynomials, it can be written as Eq. (19). Then we have

$$
\begin{aligned}
& \frac{\partial^{\alpha} u(x, t)}{\partial x^{\alpha}} \frac{\partial^{\alpha}\left(\Phi^{T}(x) \mathbf{U} \Phi(t)\right)}{\partial x^{\alpha}} \\
& =\left(\frac{\partial^{\alpha} \Phi(x)}{\partial x^{\alpha}}\right)^{T} \mathbf{U} \Phi(t) \\
& =\left(\frac{\partial^{\alpha} \Delta_{n}(x)}{\partial x^{\alpha}}\right)^{T} \mathbf{A}^{T} \mathbf{U} \Phi(t)
\end{aligned}
$$

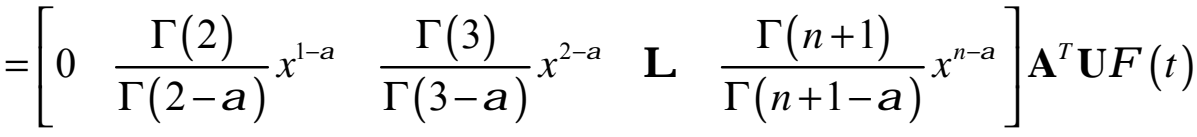

$$
\begin{aligned}
& =\left[\begin{array}{c}
1 \\
x \\
x^{2} \\
\mathrm{M} \\
x^{n}
\end{array}\right]^{T}\left[\begin{array}{ccccc}
0 & 0 & 0 & \mathrm{~L} & 0 \\
0 & \frac{\Gamma(2)}{\Gamma(2-\alpha)} x^{-\alpha} & 0 & \mathrm{~L} & 0 \\
\mathrm{M} & \mathrm{M} & \frac{\Gamma(3)}{\Gamma(3-\alpha)} x^{-\alpha} & \mathrm{L} & 0 \\
0 & 0 & \mathrm{M} & \mathrm{O} & 0 \\
0 & 0 & \mathrm{~L} & \frac{\Gamma(n+1)}{\Gamma(n+1-\alpha)} x^{-\alpha}
\end{array}\right] \mathbf{A}^{T} \mathbf{U} \Phi(t) \\
& =\Phi^{T}(x)\left(\mathbf{A}^{-1}\right)^{T} \mathbf{M} \mathbf{A}^{T} \mathbf{U} \Phi(t)
\end{aligned}
$$

and 


$$
\begin{aligned}
& \frac{\partial^{\beta} u(x, t)}{\partial t^{\beta}} \frac{\partial^{\beta}\left(\Phi^{T}(x) \mathbf{U} \Phi(t)\right)}{\partial t^{\beta}} \\
& =\Phi^{T}(x) \mathbf{U} \frac{\partial^{\beta} \Phi(t)}{\partial t^{\beta}} \\
& =\Phi^{T}(x) \mathbf{U A} \frac{\partial^{\beta} \Delta_{n}(t)}{\partial t^{\beta}}
\end{aligned}
$$

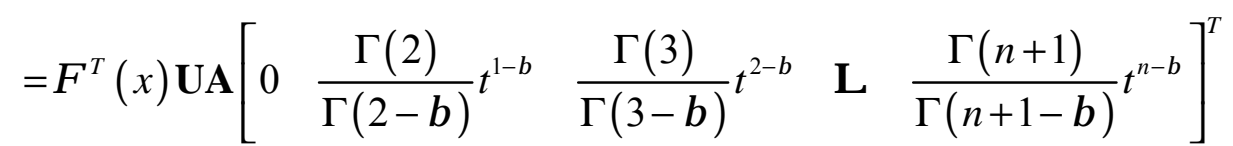

$$
\begin{aligned}
& =\Phi^{T}(x) \mathbf{U A}\left[\begin{array}{ccccc}
0 & 0 & 0 & \mathrm{~L} & 0 \\
0 & \frac{\Gamma(2)}{\Gamma(2-\beta)} t^{-\beta} & 0 & \mathrm{~L} & 0 \\
0 & 0 & \frac{\Gamma(3)}{\Gamma(3-\beta)} t^{-\beta} & \mathrm{L} & 0 \\
\mathrm{M} & \mathrm{M} & \mathrm{M} & \mathrm{O} & 0 \\
0 & 0 & 0 & \mathrm{~L} & \frac{\Gamma(n+1)}{\Gamma(n+1-\beta)} t^{-\beta}
\end{array}\right] \boldsymbol{T}_{n}(t) \\
& =\Phi^{T}(x) \mathbf{U A N A}^{-1} \Phi(t)
\end{aligned}
$$

Let

$$
\begin{aligned}
& \mathbf{M}=\left[\begin{array}{ccccc}
0 & 0 & 0 & \mathrm{~L} & 0 \\
0 & \frac{\Gamma(2)}{\Gamma(2-\alpha)} x^{-\alpha} & 0 & \mathrm{~L} & 0 \\
0 & 0 & \frac{\Gamma(3)}{\Gamma(3-\alpha)} x^{-\alpha} & \mathrm{L} & 0 \\
\mathrm{M} & \mathrm{M} & \mathrm{M} & \mathrm{O} & 0 \\
0 & 0 & 0 & \mathrm{~L} & \frac{\Gamma(n+1)}{\Gamma(n+1-\alpha)} x^{-\alpha}
\end{array}\right] \\
& \mathbf{N}=\left[\begin{array}{ccccc}
0 & 0 & 0 & \mathrm{~L} & 0 \\
0 & \frac{\Gamma(2)}{\Gamma(2-\beta)} t^{-\beta} & 0 & \mathrm{~L} & 0 \\
0 & 0 & \frac{\Gamma(3)}{\Gamma(3-\beta)} t^{-\beta} & \mathrm{L} & 0 \\
\mathrm{M} & \mathrm{M} & \mathrm{M} & \mathrm{O} & 0 \\
0 & 0 & 0 & \mathrm{~L} & \frac{\Gamma(n+1)}{\Gamma(n+1-\beta)} t^{-\beta}
\end{array}\right]
\end{aligned}
$$

Substituting Eq. (24), Eq. (25) into Eq. (1), we have

$$
a(x) \Phi^{T}(x)\left(\mathbf{A}^{-1}\right)^{T} \mathbf{M A}^{T} \mathbf{U} \Phi(t)+b(x) \Phi^{T}(x) \mathbf{U A N A}^{-1} \Phi(t)=f(x, t)
$$


Dispersing Eq. (28) by the points $\left(x_{i}, t_{j}\right)\left(i=1,2, \mathrm{~L}, n_{x} ; j=1,2, \mathrm{~L}, n_{t}\right)$, we can obtain $\mathbf{U}$ which is unknown.

\section{Numerical examples}

Example 1. Consider the following nonhomogeneous partial differential equation

$x^{1 / 3} \frac{\partial^{1 / 2} u(x, t)}{\partial x^{1 / 2}}+x^{1 / 2} \frac{\partial^{1 / 2} u(x, t)}{\partial t^{1 / 2}}=f(x, t) \quad(x, t) \in[0,1] \times[0,1]$

$u(x, 0)=10 x^{2}(1-x)$

$u(0, t)=u(1, t)=0$

where $f(x, t)=-\frac{40 \sqrt{t}(3+2 t)(-1+x) x^{7 / 3}}{3 \sqrt{\pi}}-\frac{16(1+t)^{2} x^{2}(-5+6 x)}{3 \sqrt{\pi}}$. The exact solution of the this equation is $u(x, t)=10 x^{2}(1-x)(1+t)^{2}$. Table 1-3 show the absolute errors for $t=1 / 4 s, t=1 / 2 s$, $t=3 / 4 s$ of different $n$.

Table 1.Absolute error for $t=1 / 4 s$, and different values of $n$.

\begin{tabular}{|c|c|c|c|}
\hline$x$ & $n=2$ & $n=3$ & $n=4$ \\
\hline 0.1 & 0.3246 & $3.2831 \mathrm{e}-015$ & $3.3582 \mathrm{e}-016$ \\
\hline 0.2 & 0.2351 & $4.2734 \mathrm{e}-015$ & $5.2745 \mathrm{e}-015$ \\
\hline 0.3 & 0.4820 & $4.1237 \mathrm{e}-015$ & $5.6521 \mathrm{e}-015$ \\
\hline 0.4 & 0.3274 & $5.7320 \mathrm{e}-015$ & $6.2742 \mathrm{e}-016$ \\
\hline 0.5 & 0.8231 & $4.7381 \mathrm{e}-015$ & $7.1640 \mathrm{e}-016$ \\
\hline 0.6 & 0.9127 & $7.3722 \mathrm{e}-015$ & $3.2356 \mathrm{e}-016$ \\
\hline 0.7 & 1.2188 & $6.3276 \mathrm{e}-015$ & $4.2745 \mathrm{e}-015$ \\
\hline 0.8 & 1.5181 & $1.2374 \mathrm{e}-014$ & $3.7224 \mathrm{e}-015$ \\
\hline 0.9 & 0.8364 & $3.1744 \mathrm{e}-015$ & $8.8874 \mathrm{e}-015$ \\
\hline
\end{tabular}

Table 2.Absolute error for $t=1 / 2 s$, and different values of $n$.

\begin{tabular}{|c|c|c|c|}
\hline$x$ & $n=2$ & $n=3$ & $n=4$ \\
\hline 0.1 & 0.2821 & $4.2137 \mathrm{e}-015$ & $3.4325 \mathrm{e}-016$ \\
\hline 0.2 & 0.4375 & $5.8711 \mathrm{e}-015$ & $6.5332 \mathrm{e}-016$ \\
\hline 0.3 & 0.1021 & $4.2210 \mathrm{e}-015$ & $5.2435 \mathrm{e}-016$ \\
\hline 0.4 & 0.2387 & $3.1016 \mathrm{e}-016$ & $4.9722 \mathrm{e}-016$ \\
\hline 0.5 & 0.8277 & $3.4762 \mathrm{e}-016$ & $5.3478 \mathrm{e}-015$ \\
\hline 0.6 & 0.9322 & $5.3265 \mathrm{e}-015$ & $1.3241 \mathrm{e}-016$ \\
\hline 0.7 & 1.3846 & $4.7632 \mathrm{e}-015$ & $9.2371 \mathrm{e}-016$ \\
\hline 0.8 & 1.6654 & $4.2346 \mathrm{e}-016$ & $8.4812 \mathrm{e}-015$ \\
\hline 0.9 & 0.9144 & $2.0437 \mathrm{e}-016$ & $6.3273 \mathrm{e}-016$ \\
\hline
\end{tabular}

Table 3. Absolute error for $t=3 / 4 s$, and different values of $n$. 


\begin{tabular}{|c|c|c|c|}
\hline$x$ & $n=2$ & $n=3$ & $n=4$ \\
\hline 0.1 & 0.3126 & $5.0832 \mathrm{e}-016$ & $5.3281 \mathrm{e}-016$ \\
\hline 0.2 & 0.8978 & $4.1845 \mathrm{e}-016$ & $2.3258 \mathrm{e}-015$ \\
\hline 0.3 & 0.2374 & $2.3448 \mathrm{e}-016$ & $4.0112 \mathrm{e}-016$ \\
\hline 0.4 & 0.2951 & $3.2155 \mathrm{e}-016$ & $5.1223 \mathrm{e}-016$ \\
\hline 0.5 & 0.3327 & $5.5518 \mathrm{e}-015$ & $6.3274 \mathrm{e}-015$ \\
\hline 0.6 & 1.3267 & $6.2440 \mathrm{e}-016$ & $5.7421 \mathrm{e}-015$ \\
\hline 0.7 & 0.8723 & $3.5220 \mathrm{e}-016$ & $5.2871 \mathrm{e}-016$ \\
\hline 0.8 & 0.9229 & $4.2301 \mathrm{e}-016$ & $4.8810 \mathrm{e}-016$ \\
\hline 0.9 & 1.1327 & $5.2310 \mathrm{e}-015$ & $6.1138 \mathrm{e}-016$ \\
\hline
\end{tabular}

From Tables1-3, we can see that the absolute errors are very small when $n \geq 3$. Also when $n$ is fixed, the more points we take, the more accurate numerical solutions we obtain. From Example1, we can see that the method in this paper can be effectively used to solve the numerical solution of fractional partial differential equation with variable coefficients. From the above results, the absolute errors between the numerical solutions and the exact solution are rather small. What's more, due to the absolute errors in this paper is about $10^{-15}$, Chebyshev polynomials method can reach higher degree of accuracy by comparing the approximations obtained by block pulse method [13].

\section{Summary}

In this article, we use the Chebyshev polynomials method to solve a class of fractional partial differential equation with variable coefficients. The Chebyshev polynomials operational matrix of fractional differentiation is derived from the property of Chebyshev polynomials. The initial equation is translated into the product of some relevant matrixes, which can also be regarded as the system of linear equations. The solution obtained using the suggested method is in excellent agreement with the already existing ones and shows that this approach can be solved the problem effectively. From the resulted numerical solution, we can conclude that the used techniques in this work can be applied to many other problems.

\section{References}

[1] D. Delbosco, L. Rodino, Existence and uniqueness for a nonlinear fractional differential equation. Journal of Mathmaticals Analysis and Applications, 204 (1996) 609-625.

[2] Y. Ryabov, A. Puzenko, A damped oscillations in view of the fraction oscillator equation. Physics Review B, 66 (2002) 184-201.

[3] A. M.A.EI-Sayed, Nonlinear functional differential equations of arbitrary orders. Nonliear Analysis, 33 (1998) 181-186.

[4] I. L. EI-Kalla, Error estimate of the series solution to a class of nonlinear fractional differential equations. Commun.Nonlinear Sci. Numer.Simulat., 16 (2011) 1408-1413.

[5] Z. M. Odibat, A study on the convergence of variational iteration method. Mathematical and Computer Modelling, 51 (2010) 1181-1192.

[6] S. Momani, Z. Odibat, Generalized differential transform method for solving a space and timefractional diffusion-wave equation. Physics Letters A, 370 (2007) 379-387.

[7] Z. Odibat, S. Momani, Generalized differential transform method: Application to differential equations of fractional order. Applied Mathematics and Computation, 197 (2008) 467-477.

[8] Z. Odibat, S. Momani, A generalized differential transform method for linear partial differential equations of fractional order. Applied Mathematics Letters, 21 (2008) 194-199. 
[9] Y. Zhang, A finite difference method for fractional partial differential equation, Appl. Math. Lett., 215 (2009) 524-529.

[10] Y.X. Wang, Q. B. Fan, The second kind Chebyshev wavelet method for solving fractional differential equations. Applied Mathematics and Computation, 218 (2012) 8592-8601.

[11] M. X. Yi, Y. M. Chen,: Haar wavelet operational matrix method for solving fractional partial differential equations. Computer Modeling in Engineering \&Sciences, 88(3) (2012) 229-244.

[12] Z. Odibat, S. Momani, A generalized differential transform method for linear partial differential equations of fractional order. Appl. Math. Lett., 21 (2008) 194-199.

[13] M.X. Yi, J. Huang, J.X. Wei, Block pulse operational matrix method for solving fractional partial differential equation. Applied Mathematics and Computation, 221, (2013) 121-131.

[14] I. Podlubny, Fractional Differential Equations, Academic press. 1999, pp. 121-140

[15] M.A. Snyder, Chebyshev Methods in Numerical Approximation, Prentice-Hall, Inc. Englewood Cliffs 1966, pp. 23-70.

[16] E.H. Doha, A.H. Bhrawy, S.S. Ezz-Eldien, A Chebyshev spectral method based on operational matrix for initial and boundary value problems of fractional order, Computers and Mathematics with Applications, 62 (2011) 2346-2373. 